\title{
La Función Delta de Dirac
}

\author{
Fernando Andino, Marlon Recarte, Michael Spilsbury \\ Universidad Nacional Autónoma de Honduras en el Valle de Sula
}

\begin{abstract}
La función de Dirac presenta propiedades verdaderamente útiles para modelar problemas de la física - matemática. Se presenta un resumen detallado de las principales características y propiedades de dicha "función", argumentándose la validez de las mismas.

Palabras clave: Función de Dirac, Delta de Dirac, Distribuciones
\end{abstract}

The Dirac function has properties truly useful to model problems in physics and mathematics. This document includes a detailed overview of the main characteristics and properties of the function, arguing the validity of them.

Keywords: Dirac function, Delta function, Distributions

\section{DEFINICIÓN}

$\mathrm{L}$ A función $\delta$ de Dirac no es propiamente una función, es una distribución o función generalizada (localmente integrable) introducida por primera vez por el físico ingles Paul Dirac quien la denomino como función impropia, desde el punto de vista matemático, requiere el uso de la teoría de distribuciones desarrollada por Laurent Schwartz (1940) e introducidas anteriormente de forma independiente por Sergéi Sóbolev (1935).

Puede expresarse como limite de una sucesión de funciones, lo que permite dar una idea intuitiva y operativa de la misma. Esta función constituye una aproximación muy útil para funciones pulso o función de impulso y representa igual tipo de abstracción matemática que el de una carga o masa puntual, permitiendo definir las derivadas generalizadas de funciones discontinuas.

En física esta función es muy útil ya que permite expresar magnitudes singulares en un punto como límite de magnitudes continuas, se usa para representar la distribución de densidad de una masa unidad concentrada en un punto.

La delta de Dirac es introducida para representar cierto tipo de infinitos y sus argumentos son variables reales. Para manejar estos infinitos con una notación rigurosa introducimos la cantidad $\delta$ que depende de un parámetro $x$ y satisface las condiciones:

$$
\begin{gathered}
\delta(x)= \begin{cases}0 & \text { si } x \neq 0 \\
\infty & \text { si } x=0\end{cases} \\
\int_{-\infty}^{\infty} \delta(x) d x=1
\end{gathered}
$$

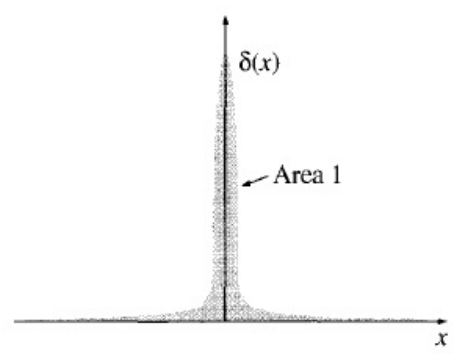

Figure 1: Representación de la delta de Dirac

Si queremos tener una imagen rigurosa de $\delta$, consideremos una función de variable real $x$ que sea nula fuera de un pequeño dominio de amplitud $\epsilon$ alrededor del origen $x=0 \mathrm{y}$ que en el interior de este dominio sea igual a uno. No importa la forma exacta de la función en el interior de este dominio, con tal que no sufra en él variaciones innecesariamente bruscas. Tomando el límite para $\epsilon \rightarrow 0$, esta función tenderá a confundirse con $\delta$. 
$\delta(x)$ no es una función de $x$ según la definición matemática ordinaria de función (que le exige tener un valor definido para cada punto de su dominio) sino algo mas general que llamaremos función impropia. Para destacar su diferencia con las funciones definidas de modo ordinario. Por tanto, $\delta(x)$ no es un cantidad que pueda usarse en análisis matemático con tanta generalidad como las funciones ordinarias, y su uso debe restringirse a cierto tipos de expresiones sencillas para las que sea evidente que no pueda dar lugar a inconsecuencias lógicas.

\section{PROPIEDADES}

La propiedad más importante de $\delta(x)$ puede expresarse con la siguiente ecuación,

$$
\int_{-\infty}^{\infty} f(x) \delta(x) d x=f(0)
$$

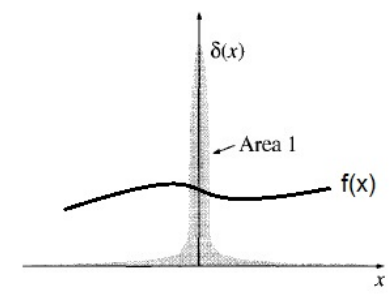

Figure 2

en la que $f(x)$ es cualquier función continua de $x$. Es fácil ver la validez de esta ecuación a partir de la imagen de $\delta(x)$ que acabamos de dar. El primer miembro de la ecuación anterior sólo puede depender de los valores muy próximos al origen, de forma que sin error notable podemos sustituir $f(x)$ por su valor en el origen $f(0)$. Esto resulta como consecuencia de la definición.

Si hacemos el cambio en el origen en la ecuación

$$
\int_{-\infty}^{\infty} f(x) \delta(x) d x=f(0)
$$

entonces podemos deducir la fórmula

$$
\int_{-\infty}^{\infty} f(x) \delta(x-a) d x=f(a)
$$

en la que $a$ es un número real cualquiera. Luego el resultado de multiplicar una función de $x$ por $\delta(x-a)$ e integrar para todo $x$ es equivalente

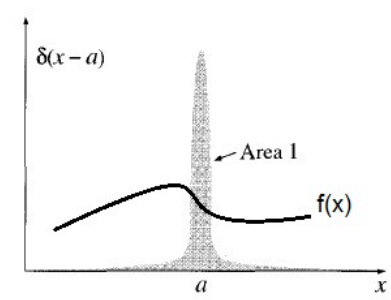

Figure 3

a sustituir $x$ por $a$. Este resultado general es válido aunque la función de $x$ no sea una función numérica, sino un vector o un operador lineal dependiente de $x$. El intervalo de integración en las fórmulas anteriores no ha de ser necesariamente de $-\infty$ a $\infty$, sino que puede ser cualquier dominio que incluya el punto crítico en el que la función $\delta$ no se anula.

Podemos definir la función $\delta$ todavía de otro modo, como la derivada $u^{\prime}(x)$ de la función $u(x)$ definida así:

$$
u(x)= \begin{cases}0 & x<0 \\ 1 & x>0\end{cases}
$$

Para comprobar que esta nueva definición es equivalente a la anterior haremos lo siguiente: Sean $a$ y $b$ dos números positivos cualquiera

$$
\int_{-a}^{b} f(x) \delta(x) d x=\int_{-a}^{b} f(x) u^{\prime}(x) d x
$$

Al integrar por partes

$$
\begin{array}{rl}
\int_{-a}^{b} & f(x) u^{\prime}(x) d x=\left.f(x) u(x)\right|_{-a} ^{b} \\
& -\int_{-a}^{b} f^{\prime}(x) u(x) d x \\
& =f(b)-\int_{0}^{b} f^{\prime}(x) d x \\
& =f(b)-[f(b)-f(0)] \\
& =f(0)
\end{array}
$$

De acuerdo a esto la función $\delta$ aparece al derivar una función discontinua.

Algunas ecuaciones elementales se pueden escribir con la función $\delta$ siendo esencialmente reglas de manipulación para desarrollos algebraicos. Estas ecuaciones dan el mismo resultado en sus dos miembros y se utilizan como factores en un integrando. 
$\boldsymbol{\delta}(-\boldsymbol{x})=\boldsymbol{\delta}(\boldsymbol{x})$ La función delta es una función par. Consideremos una función $f$ arbitraria, entonces

$$
\begin{aligned}
\int_{-\infty}^{\infty} & f(x) \delta(-x) d x \\
& =-\int_{\infty}^{-\infty} f(-x) \delta(x) d x \\
& =f(0) \\
& =\int_{-\infty}^{\infty} f(x) \delta(x) d x
\end{aligned}
$$

por lo que $\delta(-x)=\delta(x)$.

$\boldsymbol{x} \boldsymbol{\delta}(\boldsymbol{x})=\mathbf{0}$ Para probar esto sea $f(x)$ una función continua, notemos que

$$
\int f(x) x \delta(x) d x=0
$$

entonces como $f(x)$ es arbitraria el factor $x \delta(x)$ en el integrando debe ser cero.

$\delta\left(x^{2}-a^{2}\right)=\frac{1}{2 a}(\delta(x-a)+\delta(x-a))$, para $(a>0)$ Consideremos una función $f$ arbitraria, entonces

$$
\begin{aligned}
\int_{-\infty}^{\infty} & f(x) \delta\left(x^{2}-a^{2}\right) d x \\
& =\int_{-\infty}^{0} f(x) \delta\left(x^{2}-a^{2}\right) d x \\
& +\int_{0}^{\infty} f(x) \delta\left(x^{2}-a^{2}\right) d x \\
& =f(-a) \int_{-\infty}^{0} \delta\left(x^{2}-a^{2}\right) d x \\
& +f(a) \int_{0}^{\infty} \delta\left(x^{2}-a^{2}\right) d x
\end{aligned}
$$

Al hacer el cambio $z=x^{2}-a^{2}$

$$
\begin{aligned}
\int_{-\infty}^{\infty} & f(x) \delta\left(x^{2}-a^{2}\right) d x \\
& =f(-a) \int_{\infty}^{-a^{2}} \delta(z) \frac{-d z}{2 \sqrt{z+a^{2}}} \\
& +f(a) \int_{-a^{2}}^{\infty} \delta(z) \frac{d z}{2 \sqrt{z+a^{2}}} \\
& =\frac{1}{2 a} f(-a)+\frac{1}{2 a} f(a)
\end{aligned}
$$

De esto entonces

$$
\delta\left(x^{2}-a^{2}\right)=\frac{1}{2 a}(\delta(x-a)+\delta(x-a))
$$

$\int \delta(a-x) \delta(x-b)=\delta(a-b) \quad$ Este resultado puede demostrarse usando

$$
\begin{aligned}
& \int_{-\infty}^{\infty} f(x) \delta(x-a) d x=f(a), \text { tomando } \\
& f(x)=\delta(x-b), \text { tendremos }: \\
& \int_{-\infty}^{\infty} \delta(x-b) \delta(x-a) d x=\delta(a-b)
\end{aligned}
$$

Derivada de la función delta Para una función continua $f$ y un número $a \neq 0$ tenemos que

$$
\begin{aligned}
\int_{-\infty}^{\infty} & f(t)\left[\frac{\delta(t+a)-\delta(t)}{a}\right] d t \\
& =\frac{1}{a}\left[\int_{-\infty}^{\infty} f(t) \delta(t+a) d t\right. \\
& \left.-\int_{-\infty}^{\infty} f(t) \delta(t) d t\right] \\
& =\frac{1}{a}[f(-a)-f(0)]
\end{aligned}
$$

Si $a \rightarrow 0$ entonces

$$
\begin{aligned}
\lim _{a \rightarrow 0} & \int_{-\infty}^{\infty} f(t)\left[\frac{\delta(t+a)-\delta(t)}{a}\right] d t \\
& =\lim _{a \rightarrow 0} \frac{1}{a}[f(-a)-f(0)] \\
& =-f^{\prime}(0)
\end{aligned}
$$

Por lo que

$$
\int_{-\infty}^{\infty} f(x) \delta^{\prime}(x) d x=-f^{\prime}(0)
$$

El resultado anterior también puede probarse usando integración por partes

$$
\begin{gathered}
\int_{-\infty}^{\infty} f(x) \delta^{\prime}(x) d x=\left.\frac{1}{1} f(x) \delta(x)\right|_{-\infty} ^{\infty} \\
\quad-\int_{-\infty}^{\infty} f^{\prime}(x) \delta(x) d x \\
=-f^{\prime}(0)
\end{gathered}
$$

Este resultado también puede generalizarse

$$
\int_{-\infty}^{\infty} f(x) \delta^{(n)}(x) d x=(-1)^{n} f^{(n)}(0)
$$


$\delta[f(x)]=\sum_{i} \frac{1}{\left|\frac{d f}{d x}\left(x_{i}\right)\right|} \delta\left(x-x_{i}\right) \quad$ Si $f(x)$ es una función continua con $x_{i}$ raíces tal que $f\left(x_{i}\right)=0$. Sea $g(x)$ una función arbitraria, entonces

$$
\int_{-\infty}^{\infty} g(x) \delta[f(x)] d x
$$

Tomando la serie de Taylor de la función $f(x)$ alrededor de la raíz $x_{i}$ tenemos:

$$
\begin{aligned}
f(x) & =f\left(x_{i}\right)+\frac{f^{\prime}\left(x_{i}\right)}{1 !}\left(x-x_{i}\right) \\
& +\frac{f^{\prime \prime}\left(x_{i}\right)}{2 !}\left(x-x_{i}\right)^{2}+\cdots \\
& =\sum_{n=0}^{\infty} \frac{f^{n}\left(x_{i}\right)}{n !}\left(x-x_{i}\right)^{n}
\end{aligned}
$$

Donde el termino $f\left(x_{i}\right)=0 \mathrm{y}$ los términos $\left(x-x_{i}\right)^{n}$ de orden $n>1$ son despreciables, quedando únicamente el término $f^{\prime}\left(x_{i}\right)(x-$ $\left.x_{i}\right)$, por lo cual

$$
\begin{aligned}
\int_{-\infty}^{\infty} g(x) \delta[f(x)] d x \\
\quad=\sum_{i} \int_{-\infty}^{\infty} g(x) \delta\left[f^{\prime}\left(x_{i}\right)\left(x-x_{i}\right)\right] d x \\
\quad=\int_{-\infty}^{\infty} g(x) \sum_{i}\left[\frac{1}{\left|f^{\prime}\left(x_{i}\right)\right|} \delta\left(x-x_{i}\right)\right] d x
\end{aligned}
$$

\section{APLICACIONES}

\section{Representaciones}

Los siguientes tres ejemplos constituyen representaciones de la delta de Dirac.

$$
\begin{gathered}
\delta(x)=\lim _{n \rightarrow \infty} \frac{n}{\sqrt{\pi}} e^{-n^{2} x^{2}} \\
\delta(x)=\lim _{n \rightarrow \infty} \frac{\sin (n x)}{\pi x} \\
\delta(x)=\lim _{n \rightarrow \infty} \frac{n}{\pi} \cdot \frac{1}{1+n^{2} x^{2}}
\end{gathered}
$$

Todas ellas satisfacen

$$
\lim _{n \rightarrow \infty} \int_{-\infty}^{\infty} f_{n}(x) g(x)=g(0)
$$

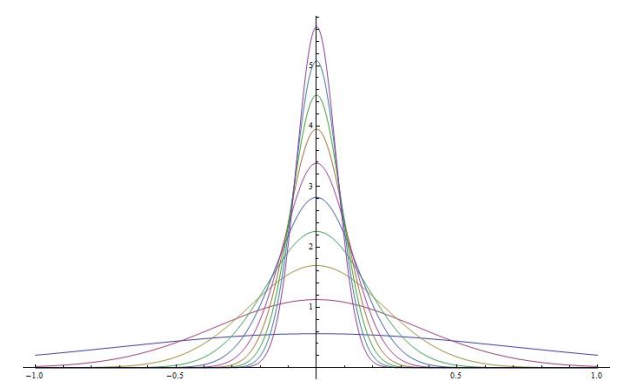

Figure 4

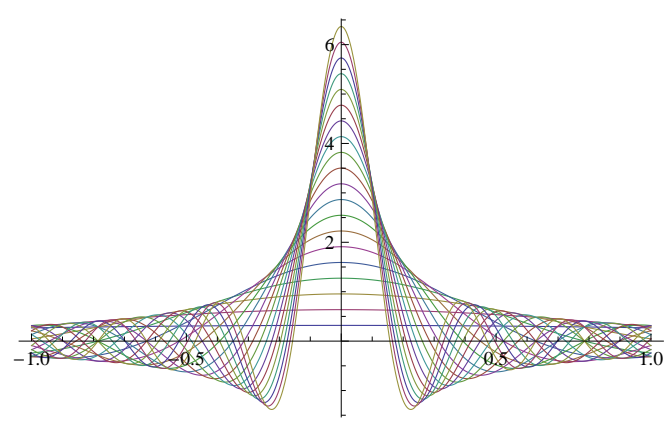

Figure 5

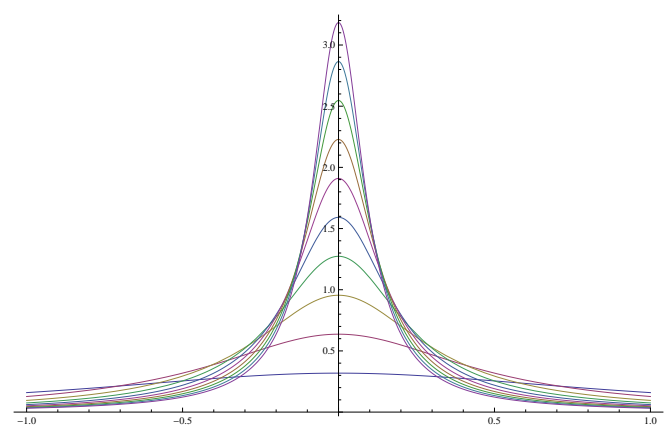

Figure 6 
Es importante tener presente que en el uso de las representaciones, las integrales se realizan primero y luego se toma el límite $n \rightarrow \infty$. El orden no conmuta.

En muchas aplicaciones de la física nos encontraremos con las siguientes representaciones equivalentes de la delta:

$$
\begin{aligned}
\delta(x) & =\lim _{n \rightarrow \infty} \frac{\sin (n x)}{\pi x} \\
& =\lim _{n \rightarrow \infty} \frac{1}{2 \pi} \int_{-n}^{n} e^{i x t} d t \\
& =\frac{1}{2 \pi} \int_{-\infty}^{\infty} e^{i x t} d t \\
\delta\left(x-x^{\prime}\right) & =\frac{1}{2 \pi} \int_{-\infty}^{\infty} e^{i\left(x-x^{\prime}\right) t} d t \\
\delta\left(x-x^{\prime}\right) & =\lim _{n \rightarrow \infty} \frac{\sin \left(n\left(x-x^{\prime}\right)\right)}{\pi\left(x-x^{\prime}\right)} \\
\delta\left(x-x^{\prime}\right) & =\lim _{n \rightarrow \infty}\left(\frac{n}{\pi} \cdot \frac{1}{1+n^{2}\left(x-x^{\prime}\right)^{2}}\right)
\end{aligned}
$$

II. Derivada de la función delta

Notemos el comportamiento de las derivadas de las representaciones de la función delta

$$
\lim _{n \rightarrow \infty} \frac{d}{d x}\left(\frac{n}{\sqrt{\pi}} e^{-n^{2} x^{2}}\right)
$$

Ver figura 7

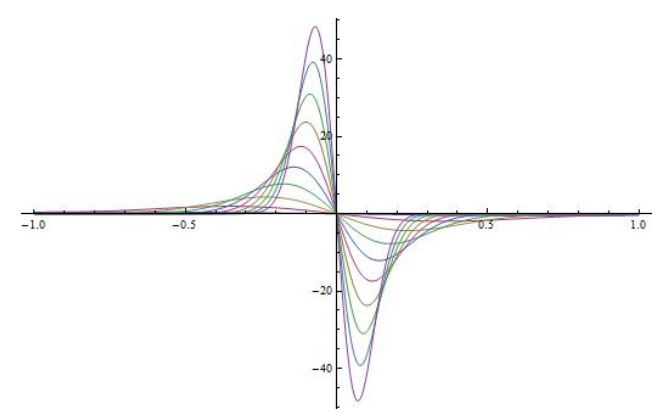

Figure 7

$$
\lim _{n \rightarrow \infty} \frac{d}{d x}\left(\frac{\sin (n x)}{\pi x}\right)
$$

Ver figura 8

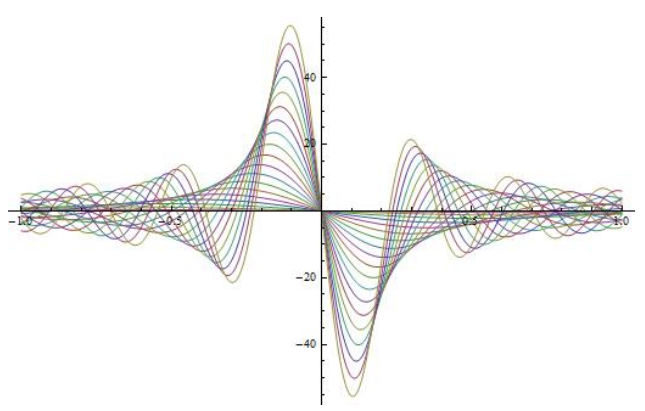

Figure 8

$$
\lim _{n \rightarrow \infty} \frac{d}{d x}\left(\frac{n}{\pi} \cdot \frac{1}{1+n^{2} x^{2}}\right)
$$

Ver figura 9

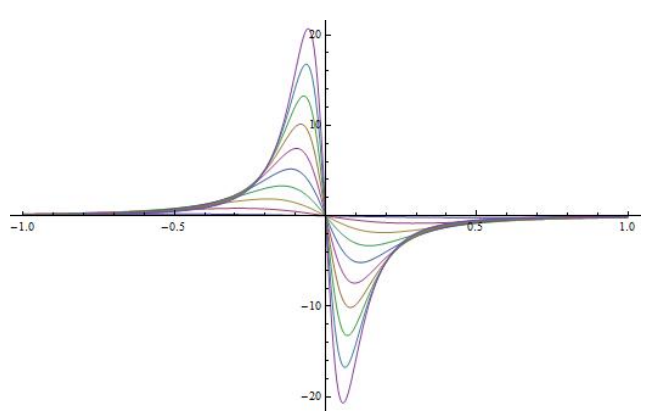

Figure 9

III. Representaciones utilizando funciones ortogonales

Si $\vec{e}(x)$ es ortogonal a $\vec{e}\left(x^{\prime}\right)$ entonces

$$
\begin{aligned}
\delta & \left(x-x^{\prime}\right)=\boldsymbol{e}(x) \cdot \boldsymbol{e}\left(x^{\prime}\right) \\
& =\sum_{n}\left(\frac{\sqrt{w(x) w\left(x^{\prime}\right)}}{E_{n}^{2}} \Psi_{n}(x) \Psi_{n}^{*}\left(x^{\prime}\right)\right)
\end{aligned}
$$

donde

$$
E_{n}^{2}=\int_{I} \Psi_{n}^{2}(x) w(x) d x
$$

En la siguiente tabla se presentan algunos polinomios ortogonales y sus características

$$
\delta\left(x-x^{\prime}\right)=\sum_{k=0}^{\infty}\left(k+\frac{1}{2}\right) P_{k}(x) P_{k}\left(x^{\prime}\right)
$$

Si consideramos $x^{\prime}=0$ entonces 
Table 1: Usando los polinomios de Legendre

\begin{tabular}{|l|l|l|l|l|}
\hline$\Psi_{n}$ & Nombre & $\mathrm{I}$ & $w(x)$ & $E_{n}^{2}$ \\
\hline$H_{n}$ & Hermite & $(-\infty, \infty)$ & $e^{-x^{2}}$ & $\sqrt{\pi} 2^{n} n !$ \\
\hline$L_{n}$ & Laguerre & {$[0, \infty)$} & $e^{-x}$ & 1 \\
\hline$P_{n}$ & Legendre & {$[-1,1]$} & 1 & $\frac{2}{2 n+1}$ \\
\hline
\end{tabular}

$$
\delta(x)=\sum_{k=0}^{\infty}\left(k+\frac{1}{2}\right) P_{k}(x) P_{k}(0)
$$

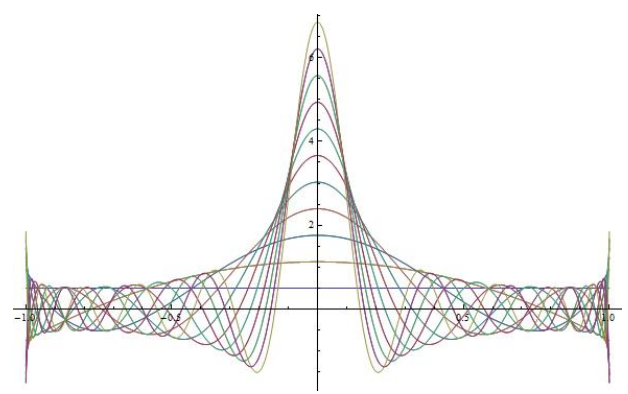

Figure 10

Usando los esféricos armónicos

$$
\begin{aligned}
\delta & \left(\cos \left(\theta_{1}\right)-\cos \left(\theta_{2}\right)\right) \delta\left(\phi_{1}-\phi_{2}\right) \\
& =\sum_{l=0}^{\infty} \sum_{m=-l}^{l} Y_{l, m}\left(\theta_{1}, \phi_{1}\right) Y_{l, m}^{*}\left(\theta_{2}, \phi_{2}\right)
\end{aligned}
$$

IV. Delta de Dirac tridimensional

Es simple generalizar la función delta en tres dimensiones

$$
\delta^{3}(\vec{r})=\delta(z) \delta(y) \delta(z)
$$

donde $\vec{r}=x \hat{x}+y \hat{y}+z \hat{z}$ es el vector de posición. La función delta tridimensional es cero en todo punto excepto en $(0,0,0)$. Su integral de volumen es:

$$
\begin{aligned}
& \int_{\mathbb{R}^{3}} \delta^{3}(\vec{r}) d \tau \\
& \quad=\int_{-\infty}^{\infty} \int_{-\infty}^{\infty} \int_{-\infty}^{\infty} \delta(x) \delta(y) \delta(z) \\
& \quad=1
\end{aligned}
$$

Además generalizando

$$
\int_{\mathbb{R}^{3}} f(\vec{r}) \delta^{3}(\vec{r}-\vec{a}) d \tau=f(\vec{a})
$$

Si consideremos un sistema coordenado curvilíneo con coordenadas $\left(\xi_{1}, \xi_{2}, \xi_{3}\right)$ y factor de escala

$$
h_{i}=\left[\left(\frac{\partial x}{\partial \xi_{i}}\right)^{2}+\left(\frac{\partial y}{\partial \xi_{i}}\right)^{2}+\left(\frac{\partial z}{\partial \xi_{i}}\right)^{2}\right]^{1 / 2}
$$

Podemos expresar la función delta

$\delta\left(\vec{r}-\overrightarrow{r_{0}}\right)=\frac{\delta\left(\xi_{1}-\xi_{10}\right)}{h_{1}} \frac{\delta\left(\xi_{2}-\xi_{20}\right)}{h_{2}} \frac{\delta\left(\xi_{3}-\xi_{30}\right)}{h_{3}}$

V. Coordenadas esféricas

$$
\begin{aligned}
\xi_{1} & =r, \xi_{2}=\theta, \xi_{3}=\phi \\
x & =r \sin (\theta) \cos (\phi), \\
y & =r \sin (\theta) \sin (\phi), \\
z & =r \cos (\theta)
\end{aligned}
$$

$$
\begin{aligned}
& h_{1}=1, h_{2}=r, h_{3}=r \sin (\theta), \\
& d v=r^{2} \sin (\theta) d r d \theta d \phi
\end{aligned}
$$

Por lo que

$$
\begin{aligned}
& \delta\left(\vec{r}-\overrightarrow{r_{0}}\right) \\
& =\frac{1}{r^{2} \sin \theta} \delta\left(r-r_{0}\right) \delta\left(\theta-\theta_{0}\right) \delta\left(\phi-\phi_{0}\right)
\end{aligned}
$$

Si se tiene simetría azimutal

$$
\delta\left(\vec{r}-\overrightarrow{r_{0}}\right)=\frac{1}{2 \pi r^{2} \sin \theta} \delta\left(r-r_{0}\right) \delta\left(\theta-\theta_{0}\right)
$$

Si no hay dependencia de $\phi, \theta$

$$
\delta\left(\vec{r}-\overrightarrow{r_{0}}\right)=\frac{1}{4 \pi r} \delta\left(r-r_{0}\right)
$$

VI. Coordenadas cilíndricas

$$
\xi_{1}=\rho, \xi_{2}=\phi, \xi_{3}=z
$$

$$
h_{1}=1, h_{2}=\rho, h_{3}=1, d v=\rho d \rho d \phi d z
$$


La correspondiente función de Dirac es

$$
\begin{gathered}
\delta\left(\vec{r}-\overrightarrow{r_{0}}\right)=\frac{1}{\rho} \delta\left(\rho-\rho_{0}\right) \delta\left(\phi-\phi_{0}\right) \delta\left(z-z_{0}\right) \\
\delta\left(\vec{r}-\overrightarrow{r_{0}}\right)=\frac{1}{2 \pi \rho} \delta\left(\rho-\rho_{0}\right) \delta\left(z-z_{0}\right) \\
\delta\left(\vec{r}-\overrightarrow{r_{0}}\right)=\frac{1}{2 \pi \rho} \delta\left(\rho-\rho_{0}\right)
\end{gathered}
$$

VII. Divergencia de $\frac{\hat{r}}{r^{2}}$

Consideremos la función vectorial $\vec{v}=\frac{\hat{r}}{r^{2}}$. Al calcular la divergencia de $\vec{v}$ obtenemos

$$
\nabla \cdot \vec{v}=\frac{1}{r^{2}} \frac{\partial}{\partial r}\left(r^{2} \frac{1}{r^{2}}\right)=\frac{1}{r^{2}} \frac{\partial}{\partial r}(1)=0
$$

Por otro lado si calculamos el flujo de $V$ sobre una esfera de radio $R$ centrada en el origen tenemos

$$
\oint \vec{v} \cdot d \vec{a}=\int\left(\frac{\hat{r}}{R^{2}}\right) \cdot\left(R^{2} \sin (\theta) d \theta d \phi \hat{r}\right)=4 \pi
$$

Usando lo anterior y el teorema de la divergencia se tiene una paradoja

$$
\int \nabla \cdot \vec{v} d \tau=\oint \vec{v} \cdot d \vec{a}
$$

Por lo que podemos definir

$$
\nabla \cdot\left(\frac{\hat{r}}{r^{2}}\right)=4 \pi \delta^{3}(\vec{r})
$$

Además

$$
\nabla\left(\frac{1}{r}\right)=-\frac{\hat{r}}{r^{2}}
$$

Entonces

$$
\nabla^{2}\left(\frac{1}{r}\right)=-4 \pi \delta^{3}(\vec{r})
$$

\section{REFERENCES}

[1] Arfken and Weber. Mathematical Methods for Physicists. Elseiver Academic Press, 2005.

[2] Paul Dirac. Principios de mecánica cuántica. Ediciones Ariel, 1958.

[3] David J. Griffiths. Introduction to Quantum Mechanics. Pearson Prentice Hall, 2005 .

[4] Hoskins. Delta Functions Introduction to Generalised Functions. Woodhead Publishing, 2009.

[5] Jackson. Classical electrodynamics. John Wiley and Sons, 1962.

[6] Morse and Feshbach. Methods of Theoretical Physics Vol 1. McGraw Hill, 1953. 